\title{
MicroRNAs regulate vascular smooth muscle cell functions in atherosclerosis (Review)
}

\author{
$\mathrm{XIN} \mathrm{YU}^{1,2^{*}}$ and $\mathrm{ZHENG} \mathrm{LI}^{1 *}$ \\ ${ }^{1}$ Department of Orthopaedic Surgery, Peking Union Medical College Hospital, Peking Union Medical College, Beijing 100730; \\ ${ }^{2}$ State Key Laboratory of Cardiovascular Disease, Fuwai Hospital, National Center for Cardiovascular Diseases, \\ Chinese Academy of Medical Sciences and Peking Union Medical College, Beijing 100037, P.R. China
}

Received December 31, 2013; Accepted May 30, 2014

DOI: $10.3892 /$ ijmm.2014.1853

\begin{abstract}
Vascular smooth muscle cells (VSMCs) are involved in all stages of the progression of human atherosclerosis (AS). MicroRNAs (miRNAs or miRs) are non-coding, small RNAs that regulate gene expression at the post-transcriptional level through translational repression or messenger RNA (mRNA) decay. Recently, a variety of functions of VSMCs that are involved in AS, including differentiation, migration, proliferation, extracellular matrix (ECM) synthesis and apoptosis, have been found to be regulated by miRNAs. This review provides an overview of the role of miRNAs in the regulation of the functions of VSMCs, as well as their targets and potential implications in AS. The data presented herein suggest that the specific modulation of miRNAs may present an attractive approach for the diagnosis and treatment of AS.
\end{abstract}

\section{Contents}

\section{Introduction}

2. miRNAs regulate VSMC differentiation and phenotype

3. miRNAs and pro-contractile phenotype

4. miRNAs and pro-synthetic phenotype

5. miRNAs regulate VSMC proliferation

6. miRNAs promote the proliferation of VSMCs

7. miRNAs inhibit the proliferation of VSMCs

8. miRNAs regulate VSMC migration

9. miRNAs promote the migration of VSMCs

10. miRNAs inhibit the migration of VSMCs

Correspondence to: Dr Zheng Li, Department of Orthopaedic Surgery, Peking Union Medical College Hospital, No. 1 Shuaifuyuan, Dongcheng, Beijing 100730, P.R. China

E-mail:kleeo@163.com

*Contributed equally

Key words: vascular smooth muscle cells, microRNAs, atherosclerosis, restenosis
11. miRNAs regulate VSMC apoptosis

12. miRNAs regulate ECM synthesis by VSMCs

13. miRNAs regulate VSMC calcification

14. Implications in the management of AS

15. Conclusions

\section{Introduction}

Cardiovascular disease (CVD) of atherosclerotic origin remain the leading cause of mortality worldwide, accounting for approximately $27 \%$ of deaths among males and $32 \%$ among females. The initiation and progression of atherosclerosis (AS) involves the chronic expansion of arterial intima with diverse types of cells, including macrophages, dendric cells (DCs), vascular smooth muscle cells (VSMCs) and endothelial cells (ECs), as well as various sets of pro-inflammatory substances, such as adhesion molecules, cytokines, chemotactic factors and extracellular matrix (ECM) proteins (1).

VSMCs are the most abundant cell type in the arterial wall and are involved in the progression of AS. VSMCs exist in many phenotypes $(2,3)$. Under normal conditions, the predominant phenotype is quiescent/contractile with non-migratory and non-proliferative VSMCs. The loss of ECs due to mechanical forces, including hemodynamic forces and stenting or cell apoptosis, can lead to VSMC migration from the media to the intima, where VSMCs can proliferate to form the neointima and plaque (4-6). VSMCs contribute to the development of AS through a variety of functions, including migration, proliferation, ECM synthesis and apoptosis, as well as inflammation and foam cell formation through cholesterol uptake (7).

MicroRNAs (miRNAs, miRs) are small, non-coding RNAs that can control gene expression by binding to their target messenger RNAs (mRNAs) through translational repression or mRNA decay (8). By binding to target genes and directly degradating mRNA or repressing translation, miRs are able to regulate the expression of hundreds or thousands of genes. The regulation of miRs is involved in a number of physiological and pathological conditions, such as metabolism, differentiation, development, apoptosis, proliferation and oncogenesis (9).

Recent studies have found that miRs play an important role in vascular pathophysiology and AS (10-12). Abnormal miR expression profiles have been reported to be related to CVD in 
Table I. miRs involved in the regulation of the VSMC phenotype.

\begin{tabular}{|c|c|c|c|}
\hline Function & miRs & Targets & (Refs.) \\
\hline $\begin{array}{l}\text { Promote contractile } \\
\text { phenotype }\end{array}$ & $\begin{array}{l}\text { miR-1 } \\
\text { miR-31 } \\
\text { miR-133 } \\
\text { miR-143/145 }\end{array}$ & $\begin{array}{l}\text { KLF4, Pim-1 } \\
\text { CREG } \\
\text { Sp-1, moesin } \\
\text { ELK1, FRA1 } \\
\text { ACE, KLF4/5, } \\
\text { CALMK, MRTF-B }\end{array}$ & $\begin{array}{c}(19-21) \\
(29) \\
(3) \\
(35,40)\end{array}$ \\
\hline $\begin{array}{l}\text { Promote synthetic } \\
\text { phenotype }\end{array}$ & $\begin{array}{l}\operatorname{miR}-24 \\
\text { miR-26a } \\
\text { miR-221/222 }\end{array}$ & $\begin{array}{l}\text { Trb3 } \\
\text { SMAD1/4 } \\
\text { c-kit, p27 (Kip1) } \\
\text { p57 (Kip2) }\end{array}$ & $\begin{array}{l}(41) \\
(43) \\
(46)\end{array}$ \\
\hline Dual effect & miR-21 & PDCD4, Sp-1 & $(50-52)$ \\
\hline
\end{tabular}

over 400 studies (e.g., 13). Moreover, accumulating evidence suggests a close association between miRs and the functions of VSMCs (14). The identification of the role of miRs in the regulation of VSMCs may lead to an improved in the understanding of the pathophysiology of AS and is likely to provide novel diagnostic and therapeutic targets.

In this review, we summarize the current knowledge on the role of miRs in the regulation of VSMC functions, including differentiation, proliferation, ECM synthesis, apoptosis and calcification. In particular, we discuss the mechanisms of action of miRs in the regulation of VSMC functions and their possible implications in AS. In addition, we discuss the potential applicability of miRs in the diagnosis and treatment of AS.

\section{2. miRNAs regulate VSMC differentiation and phenotype}

VSMCs can switch between a differentiated (contractile) state and a dedifferentiated (synthetic) phenotype in response to extracellular stimulation. Spindle-shaped differentiated VSMCs have low rates of proliferation, migration and production of ECM, but high levels of contractile genes, while rhomboid-shaped dedifferentiated VSMCs have increased rates of proliferation, migration and production of ECM, as well as low levels of contractile genes (15). Under pathological conditions, VSMCs can undergo the phenotypic change from the normal contractile state to synthetic, proliferative cells, which have an increased ability to migrate, proliferate and produce ECM (16). In atherosclerotic lesions and neointimal tissue, synthetic VSMC phenotypes are the major cellular types in vascular repair following various injuries. The phenotypic switching of VSMCs depends on numerous local signals, such as cytokines, cell-cell contact, cell adhesion, ECM interactions, injury stimuli, among which platelet-derived growth factor (PDGF)-BB and transforming growth factor- $\beta$ (TGF- $\beta$ )/bone morphogenetic protein (BMP) are the key mediators of VSMC phenotypic switching (17). PDGF-BB significantly promotes the VSMC phenotype switch from a differentiated, contractile state into a dedifferentiated, synthetic state, thus promoting VSMC proliferation and migration, while TGF- $\beta$ and BMP help to maintain the VSMC contractile phenotype $(18,19)$. Abnormal regulation of this phenotype switching leads to the development and progression of AS and restenosis (20). By preventing the VSMC phenotypic modulation from a contractile state to a synthetic state, neointimal progression in AS may be controlled. Previous studies have identified many miRs in the regulation of VSMCs phenotypic modulation (Table I and Fig. 1).

\section{3. miRNAs and pro-contractile phenotype}

miR-1 levels are significantly higher in more differentiated and less proliferative VSMCs and downregulated during neointima formation (21). miR-1 has been proven to regulate the differentiation of VSMCs by targeting Krüppel-like factor 4 (KLF4) and proviral integration site for Moloney murine leukemia virus 1 (Pim-1) (21-23). KLFs are a subclass of evolutionarily conserved transcription factors (24), and KLF4 has been demonstrated to significantly repress the expression of multiple genes in VSMCs during the phenotypic switching of SMCs in response to vascular injury, TGF- $\beta$ or PDGF-BB (25-27). For instance, KLF4 can downregulate the dedifferentiation marker gene, non-muscle myosin heavy chain B (Smemb) (28). Pim-1, an oncogenic serine/threonine kinase, has been reported to induce neointimal hyperplasia formation and promote the proliferation of cultured VSMCs (29).

miR-31 is upregulated in contractile VSMCs and downregulated in proliferative VSMCs, the proliferation of which was induced by PDGF (58). In addition, miR-31 levels have been shown to be higher in the serum of patients with coronary artery disease (CAD) with restenosis compared to patients with CAD without restenosis (30). miR-31 has been proven to promote the VSMC contractile phenotype by repressing cellular repressor of E1A-stimulated genes (CREG) expres- 


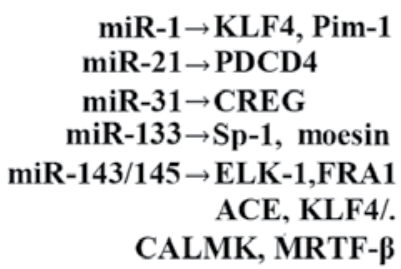

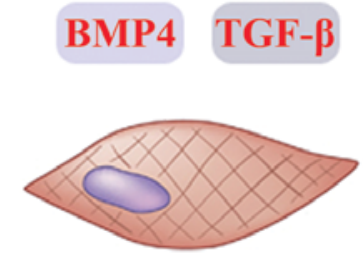
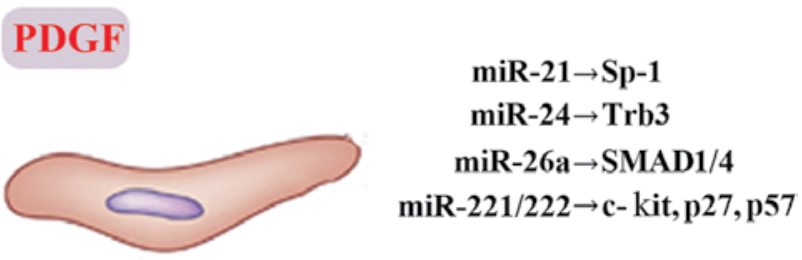

Differentiated, contractile SMCs $\downarrow$ proliferation, $\downarrow$ migration
De-differentiated, synthetic SMCs $\uparrow$ proliferation, $\uparrow$ migration

Figure 1. Regulation of vascular smooth muscle cell (VSMC) proliferation and migration by microRNAs (miRs) and their targets. miR-1, miR-21, miR-31, miR-133 and miR-143/145 inhibit bone morphogenetic protein (BMP) 4 and transforming growth factor- $\beta$ (TGF- $\beta$ )-induced VSMC proliferation and migration. miR-21, miR-24, miR-26, and miR-221/222 increase PDGF-induced VSMC proliferation and migration.

sion. It has been reported that the overexpression of CREG maintains the differentiated phenotype in cultured VSMCs, whereas shRNA-mediated CREG knockdown promotes VSMC dedifferentiation, implicating a critical role for CREG in maintaining the phenotypic switching of VSMCs (31). However, the exact mechanism of action of CREG in regulating VSMC phenotypic modulation remains unclear.

miR-133 is downregulated in proliferating VSMCs but upregulated when VSMCs are back to the quiescence phenotype (32). Furthermore, the circulating levels of miR-133 are also downregulated in humans with CAD (33). miR-133 has been shown to inhibit the VSMC switch to the synthetic phenotype by inhibiting the tracription factor, specificity protein-1 (Sp-1) (32). Sp-1 is a transcription factor activated by a phenotypic switch that promotes stimuli and is upregulated in animal models of vascular injury $(34,35)$. Activated $\mathrm{Sp}-1$ in turn increases the activity of KLF4, which can repress myocardin and therefore downregulates the expression of most of the genes in VSMCs (36).

miR-143/145, encoded by a bicistronic gene cluster, are highly conserved and abundantly expressed in normal VSMCs, regulating VSMC differentiation. miR-143/145 are downregulated in animal models of AS $(37,38)$. Furthermore, the circulating levels of miR-143/145 are downregulated in humans with CAD (33). The transfer of miR-143 and miR-145 from ECs to VSMCs promotes the contractile VSMC phenotype and attenuates AS (39). Aged miR-143/145 ${ }^{-/-}$mice develop spontaneous atherosclerotic lesions in the femoral artery in the absence of hypercholesterolemia, and the overexpression of miR-145 reduces AS in Apoe ${ }^{-/-}$mice $(40,41)$. A network of transcription factors, such as KLF4, KLF5, ELK1, versican, several actin remodeling proteins and angiotensin-converting enzyme (ACE), have been identified as the targets of miR-143/145 (37,42). These targets function as transcription and translation factors, receptors, phosphatases, kinases, growth factors, RNA binding proteins, and so forth, and are involved in different cellular processes in addition to regulating VSMC plasticity. This represents an example of how a single miRNA can regulate a number of related or unrelated cellular processes.

\section{4. miRNAs and pro-synthetic phenotype}

In response to PDGF-BB, the expression of miR-24 is upregulated and its target, Tribbles-like protein 3 (Trb3), is downregulated (43). Trb3 has been reported to interact with type-II BMP receptor (BMPRII) and promote the degradation of Smad ubiquitin-regulatory factor-1 (Smurf1), a negative regulator of BMP and TGF- $\beta$ Smad-dependent signalling (44). Trb3 has been proven to be essential for the PDGF-BBmediated induction of the VSMC synthetic phenotype. In addition, VSMC proliferation and the repression of Trb3 coincides with the reduced expression of Smad proteins and a decrease in BMP and TGF- $\beta$ signalling, which promotes a synthetic phenotype in VSMCs.

It has been demonstrated that miR-26a has multiple roles in VSMCs, including the promotion of VSMC proliferation, migration and the inhibition of apoptosis. miR-26a is significantly upregulated in synthetic VSMCs (45). The overexpression of miR-26a inhibits the differentiation of VSMCs and helps maintain the balance of cells in the synthetic and contractile states and governs phenotypic shifting by directly targeting SMAD1 and SMAD4 (45). SMAD1 and SMAD4 are two TGF- $\beta$ - and BMP-related pro-differentiation factors, in which SMAD-1 is primarily an element of BMP-responsive pathways, while SMAD-4 is in the common pathway for BMP and TGF- $\beta$ (45).

miR-221 and miR-222 are clustered on the X chromosome and share a common seed sequence. Both are significantly upregulated in vivo in VSMCs following the balloon injury of the vessel (46). miR-221 is upregulated in VSMCs upon the PDGF-BB-mediated VSMC phenotypic switch into the synthetic phenotype (47). miR-221/222 can regulate multiple functions of VSMCs, including the reduced expression of contractile genes, and increased proliferation and migration. miR-221/222 regulate multiple functions through multiple targets, including p27 (Kip1), p57 (Kip2) and c-kit (48). p27 and p57 are cyclin-dependent kinase inhibitors (CKIs), negatively regulating cell proliferation. The overexpression of $\mathrm{p} 27$ or p57 inactivates the cyclin-CDK complexes and leads to cell cycle arrest, inversely correlates with VSMC proliferation (49). 
Other than the cell cycle control, p57 has been reported to be involved in cell differentiation in chondrocytes (50). c-kit, a proto-oncogene, is involved in multiple cellular functions, such as cell survival, proliferation, differentiation, adhesion, homing and migration (51) However, to the best of our knowledge, the role of p27, p57 and c-kit in VSMC differentiation has not been investigated to date.

miR-21 regulates multiple functions of VSMCs, including phenotypic changes, proliferation and apoptosis. However, its role in the phenotypic modulation and differentiation of VSMCs remains controversial. In some studies, miR-21 has been proven to inhibit VSMC differentiation, promote proliferation and reduce apoptosis through Sp-1 $(52,53)$. However, another study demonstrated that miR-21 increases the biosynthesis of contractile protein in VSMCs by targeting programmed cell death protein 4 (PDCD4) (54). PDCD4, a tumor suppressor gene, has been reported to promote the differentiation of acute myeloid leukemia (AML) cells and female germline stem cells $(55,56)$. However, to our knowledge, the role of PDCD4 in VSMC differentiation has not been investigated to date. The dual effect of miR-21 may be due to the diverse target genes of miR-21 in regulating VSMC differentiation. Further studies are required to elucidate the exact role of miR-21 in the phenotypic regulation of VSMCs.

\section{5. miRNAs regulate VSMC proliferation}

Aberrant VSMC proliferation significantly contributes in neointimal plaque formation during the progression of AS. VSMC proliferation has been proven to be solely responsible for in-stent restenosis (57). As mentioned above, VSMCs can switch between two phenotypes, a differentiated/contractile phenotype and a dedifferentiated/synthetic phenotype. VSMCs with the dedifferentiated/synthetic phenotype have an increased ability to migrate, proliferate and produce ECM, which contributes to the development of AS. Thus, the proliferation of VSMCs is closely associated with the phenotypic regulation of VSMCs. Accumulating evidence has demonstrated that miRs play an important role in the mediation of VSMC proliferation through the regulation of the post-transcriptional expression of several genes (Table II). Some miRs have been found to promote the proliferation of VSMCs, while others have been reported to inhibit the proliferation of VSMCs. By preventing VSMC proliferation, neointimal progression in AS may be controlled.

\section{6. miRNAs promote the proliferation of VSMCs}

miR-21 is one of the most upregulated miRs in the vascular wall following balloon injury. The inhibition of miR-21 can decrease the proliferation of cultured VSMCs in injured rat carotid arteries (53).

In addtion to phenotypic regulation, miR-26a can also promote VSMC proliferation and aortic SMCs deficient in miRNA-26a show a significant reduction in proliferation (45).

As mentioned above, miR-31 can modulate the VSMC phenotype by targeting CREG (30). It can also regulate the proliferation of VSMCs. The expression of miR-31 is significantly increased in proliferative VSMCs. miR-31 exerts a pro-proliferative effect on VSMCs by targeting large tumor
Table II. miRs involved in the regulation of VSMC proliferation.

\begin{tabular}{|c|c|c|c|}
\hline Function & miRs & Targets & (Refs.) \\
\hline \multirow{7}{*}{$\begin{array}{l}\text { Promote } \\
\text { proliferation }\end{array}$} & miR-21 & PTEN, Bcl-2 & (51) \\
\hline & miR-26a & SMAD-1/4 & (43) \\
\hline & miR-31 & LATS2 & $(56)$ \\
\hline & miR-130a & GAX & (58) \\
\hline & miR-146a & KLF4 & (66) \\
\hline & miR-208 & p21 & (67) \\
\hline & miR-221/222 & $\begin{array}{l}\text { PTEN, Bcl-2, } \\
\text { p27, p57 }\end{array}$ & $(44,45)$ \\
\hline \multirow{13}{*}{$\begin{array}{l}\text { Inhibit } \\
\text { proliferation }\end{array}$} & miR-1 & Pim-1 & (13) \\
\hline & $\operatorname{miR}-15 \mathrm{a}$ & $?$ & (39) \\
\hline & miR-133 & Sp-1 & (17) \\
\hline & miR-143/145 & $\begin{array}{l}\text { KLF5, ELK1, } \\
\text { myocardin }\end{array}$ & (19) \\
\hline & miR-152 & DNMT1 & $(42)$ \\
\hline & miR-155 & - & (44) \\
\hline & miR-181a & - & $(45)$ \\
\hline & miR-195 & $\begin{array}{l}\text { Cdc42, CCND1, } \\
\text { FGF1 }\end{array}$ & $(46)$ \\
\hline & $\mathrm{miR}-424 / 322$ & $\begin{array}{l}\text { Cyclin D1, } \\
\text { calumenin }\end{array}$ & $(47)$ \\
\hline & miR-490-3p & PAPP-A & (48) \\
\hline & miR-638 & NDR1 & (49) \\
\hline & let-7d & KRAS & $(50)$ \\
\hline & let- $7 \mathrm{~g}$ & LOX-1 & $(51)$ \\
\hline
\end{tabular}

miRs, microRNAs; VSMCs, vascular smooth muscle cells; LATS2, large tumor suppressor homolog 2; GAX, growth arrest-specific homeobox, KLF4, Krüppel-like factor 4; Pim-1, proviral integration site 1 ; Sp-1, specificity protein-1; DNMT1, DNA methyltransferase 1; PAPP-A, pregnancy-associated plasma protein A; LOX-1, lectin-like oxidized low-density lipoprotein receptor-1.

suppressor homolog 2 (LATS2) (58). LATS2 has been proven to play a major role in cell proliferation and apoptosis and is an important regulator of tissue and organ development (59).

miR-130a is upregulated in the remodeled aorta and arteries with hypertension. The overexpression of miR-130a has been shown to significantly promote the proliferation of VSMCs by inhibiting the expression of growth arrest-specific homeobox (GAX) (60). GAX has been proven to be downregulated in some vascular diseases, such as balloon injury (61), pulmonary hypertension (62), or by certain growth factors including Ang-II (63) and PDGF (64). GAX can significantly inhibit the proliferation, differentiation and migration of VSMCs $(65,66)$.

The expression of miR-146a is significantly upregulated in atherosclerotic arteries compared with non-atherosclerotic arteries(38).miR-146a promotes VSMC proliferationincultured VSMCs by targeting KLF4. KLF4 exerts an anti-proliferative effect on VSMCs through the upregulation of $\mathrm{p} 21$, which is a member of the cyclin-dependent kinase (CDK)-inhibitory protein family (67). The transfection of antisense miR-146a 


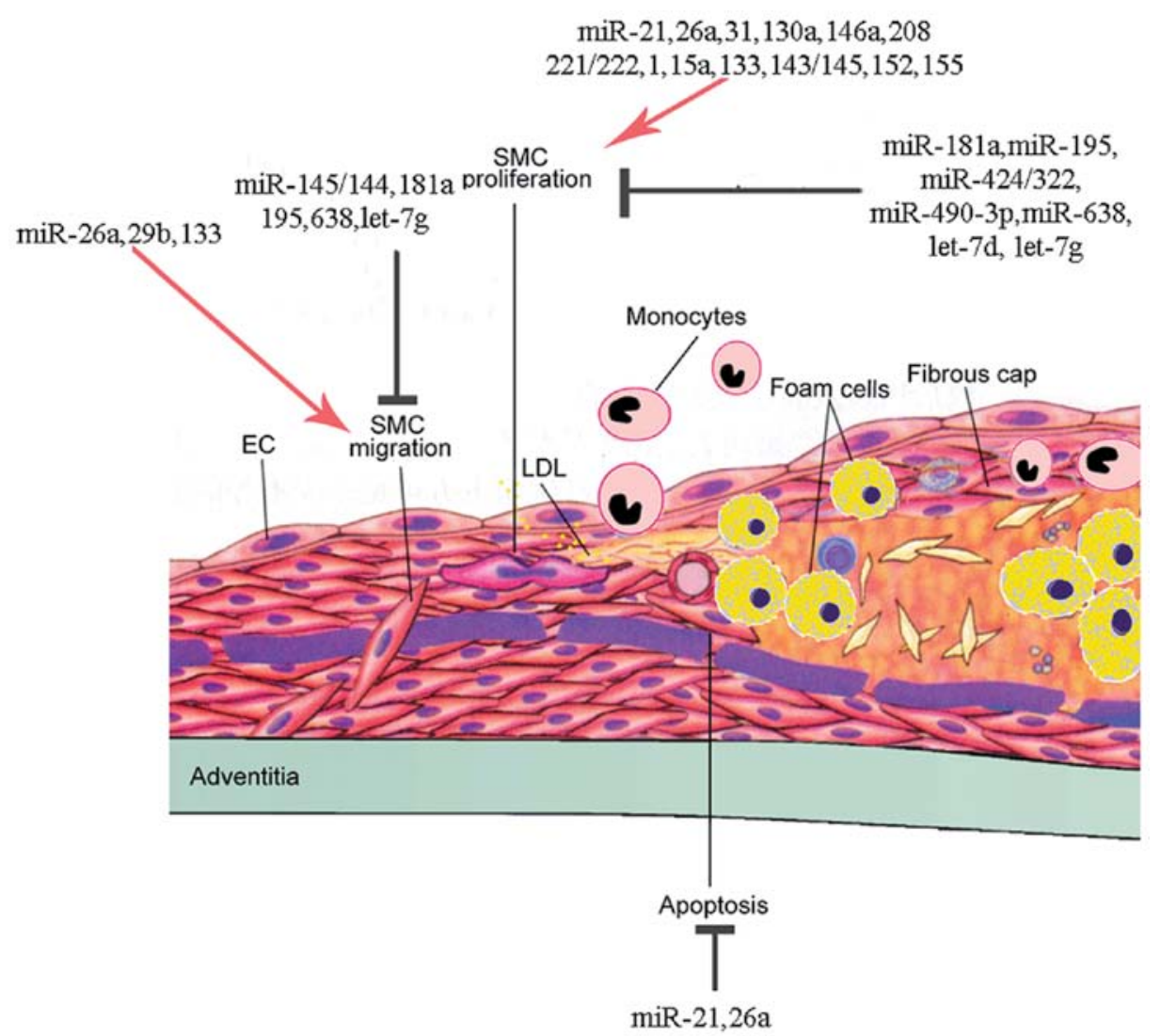

Figure 2. Regulation of vascular smooth muscle cell (VSMC) phenotypic switch by microRNAs (miRs) and their targets. VSMCs switch between the contractile phenotype and the synthetic phenotuype in response to several external stimuli. Deregulation of switching between these phenotypes is associated with vascular disorders and atherosclerosis (AS). Under normal conditions, platelet-derived growth factor (PDGF) induces multiple aspects of the synthetic VSMC phenotype, characterized by increased rates of proliferation and migration. Inversely, transforming growth factor- $\beta$ (TGF- $\beta$ ) and the related factor bone morphogenetic protein 4 (BMP 4) promote the contractile VSMC phenotype, characterized by decreased rates of proliferation and migration. miRNAs reported to modulate the VSMC phenotype switching are shown. KLF4/5, Krüppel-like factor 4/5; Pim-1, proviral integration site 1; CREG, cellular repressor of E1A-stimulated gene; SP-1, specificity protein-1 transcription factor; ELK1, ELK1 members of ETS oncogene family; FRA1, Fos-related antigene 1; ACE, angiotensin-converting enzyme; CALMK, calmodulin K; MRTF-B, myocardin related transcription factor B; Trb3, Trbbles-like protein-3; p27, cyclin-dependent kinase inhibitor 1C (CDKN1C); PDCD4, programmed cell death protein 4.

oligonucleotide into balloon-injured rat carotid arteries has been shown to markedly decrease neointimal hyperplasia (68). miR-208 regulates insulin-induced VSMC proliferation through the downregulation of its potential target, p21. However, as previously demonstrated, an miR-208 inhibitor alone had no effect on VSMC proliferation (69).

As mentioned above, miR-221/222 regulates multiple functions of VSMCs, such as reducing the expression of contractile genes and increasing proliferation and migration. The expression of miR-221/222 is significantly increased in proliferative VSMCs. The knockdown of miR-221/222 has been shown to decrease the proliferation of cultured VSMCs and neointima formation in balloon-injured rat arteries (46). miR-221 and miR-222 have been proven to regulate VSMC proliferation by targeting p27 and p57 (46,47). p27 and p57 are CKIs, negatively regulating cell proliferation (Fig. 2).

\section{7. miRNAs inhibit the proliferation of VSMCs}

As mentioned above, miR-1 expression is significantly higher in more differentiated and less proliferative VSMCs and is downregulated during neointima formation. miR-1 expression is higer in VSMCs treated with myocardin, which can strongly inhibit the proliferation of VSMCs. The introduction of miR-1 into VSMCs inhibits their proliferation by directly targeting Pim-1 (21). Pim-1 has been proven to encode an oncogenic serine/threonine kinase, which is required for injury-induced neointima formation and SMC proliferation (29). miR-15a is upregulated in VSMCs treated with KLF4, which can strongly inhibit the proliferation of VSMCs. Therefore, miR-15a contributes to the anti-proliferative behaviors of KLF4, which has a strong inhibitory effect on the proliferation of VSMCs (70).

In addition to the phenotypic regulation of VSMCs mentioned earlier, miR-133 expression is decreased in proliferating VSMCs and following vascular injury. The overexpression of miR-133 decreases VSMC proliferation and migration, while anti-miR-133 can lead to an increased proliferation and migration by specifically suppressing the expression of the transcription factor, Sp-1 (32).

As mentioned earlier, miR-143/145 expression is downregulated following both vascular injury and PDGF-BB treatment. The restoration of the expression of miR-143/145 inibits neointima formation in the rat carotid artery following 
balloon injury $(37,71,72)$. The adenovirus-mediated gene transfer of miR-145 in rat balloon-injured carotid arteries has been proven to inhibit neointimal lesion formation (71).

miR-152 is downregulated in lipopolysaccharide (LPS)treated VSMCs. The overexpression of miR-152 decreases cell proliferation in LPS-treated VSMCs. miR-152 exerts its effects by downregulating DNA methyltransferase 1 (DNMT1) and subsequently decreasing the methylation of ER $\alpha$ gene promoter region (73). DNMT1 is the most abundant DNA methyltransferase in mammalian cells and is the key maintenance enzyme for hemimethylated DNA during DNA replication of various types of cancer (74).

miR-155 is specifically expressed in atherosclerotic plaques, where it is induced by oxidized low-density lipoprotein (ox-LDL) (75). However, the circulating levels of miR-155 have been shown to be significantly downregulated in patients with CAD compared to the controls (33). Therefore, in vivo studies on the role of miR-155 in AS are conflicting. miR-155 is considered to contribute to the suppression of certain members of the matrix metalloproteinase (MMP) family (76). MMPs are involved in the atherosclerotic plaque formation by participating in ECM production and cell migration. In particular, MMPs can destruct the existing collagen that lends strength to the protective fibrous cap of atherosclerotic plaques, which may lead to lethal thrombosis and stenosis in AS.

miR-181a is downregulated by Ang-II, which plays a central role in the pathogenesis of AS. miR-181a inhibits the adhesion of VSMCs to collagen in response to Ang-II, which is essential for migration and proliferation (77). The overexpression of miR-181a inhibits Ang-II-mediated osteopontin (OPN) expression, which is a pro-inflammatory molecule and is found in abundance in atherosclerotic plaques.

miR-195 expression is altered in ox-LDL-treated VSMCs. miR-195 inhibits ox-LDL-induced VSMC proliferation, migration and synthesis the of IL-1 $\beta$, IL-6, and IL-8. In addition, the administration of miR-195 has been shown to substantially reduce neointima formation, and thus plays a potential therapeutic role (78). miR-195 can repress the expression of the Cdc42, cyclin D1 (CCND1) and fibroblast growth factor 1 (FGF1) genes, which are functionally related in the same signalling pathway and are involved in cell cycle control, migration and proliferation. $\mathrm{Cdc} 42$ is a downstream effector of phosphoinositide 3-kinase (PI3K) and can prommote CCND1 (78) and FGF1 (80) expression. FGF1 has been reported to be an important angiogenic factor that can induce VSMC proliferation and migration $(81,82)$.

miR-424, the ortholog of rat miR-322, is upregulated in proliferative VSMCs and after vascular injury. However, increased levels of miR-424/322 inhibit VSMC proliferation in vitro and in injury-induced remodeling in vivo (83). In addition, the early and ample introduction of miR-424/322 in vivo in balloon-injured vessels has been shown to markedly reduce neointima formation in a rat model of restenosis. Cyclin D1 and calumenin have been confirmed to be the direct target genes of miR-424/322. Cyclin D1 has been proven to be a direct target of miR-424 in other tissues (84). It has been demonstrated that calumenin, a $\mathrm{Ca}^{2+}$-binding protein, is a regulator of SERCA2a, which is able to inhibit its activity in cardiac myocytes (85). miR-424/322 can inhibit calumenin protein expression. This decrease can improve SERCA2a activity and allow better $\mathrm{Ca}^{2+}$ cycling in VSMCs.
miR-490-3p is significantly downregulated in in ox-LDLtreated VSMCs and human AS plaques. miR-490-3p inhibits the proliferation of VSMCs induced by ox-LDL by targeting pregnancy-associated plasma protein A (PAPP-A) (86). PAPP-A, a metalloproteinase, is abundantly expressed in VSMCs. PAPP-A can activate local insulin-like growth factor (IGF). However, whether PAPP-A is pro- or anti-atherosclerotic protein remains controversial since the role of IGF in AS has not yet been fully elucidated.

miR-638, which is enriched in human aortic VSMCs, is significantly downregulated in proliferative VSMCs. miR-638 inhibits PDGF-BB-induced VSMC proliferation and migration by targeting neuron-derived orphan receptor 1 (NOR1) (87). NOR1 is a critical regulator implicated in proliferative vascular diseases (88).

Let-7d is downregulated in proliferating VSMCs. The overexpression of let-7d in VSMCs reduces VSMC growth by targeting KRAS (89). KRAS, a member of the small GTPase superfamily, regulates the pathway involved in proliferation, differentiation and programmed cell death $(90,91)$.

The human serum levels of let-7g are lower in subjects with hypercholesterolemia compared with normal controls. A lower level of let-7g has been observed in mice fed a highfat diet than in mice fed a normal chow diet. The tansfection of let-7g into VSMCs has been shown to significantly inhibit VSMC proliferation and migration induced by ox-LDL by targeting lectin-like oxidized-low-density lipoprotein receptor-1 (LOX-1) (92). The role of LOX-1 in the pathogenesis of vascular disorders has been well documented (93). LOX-1 can be activated by oxLDL and stimulates the expression of endothelial pro-inflammatory genes and superoxide radical formation, which is believed to play an active role in atherogenesis (94) (Fig. 2).

\section{8. miRNAs regulate VSMC migration}

In the native vessel, VSMCs are maintained in a quiescent/contractile and non-migratory and non-proliferative state, surrounded by a complex, highly structured ECM. In response to vascular injury, such as platelet aggregation on the lining of the vascular wall, or mechanical injury (ballooning with or without stenting, VSMCs can migrate from the medial wall to the intimal space, where they can proliferate and deposit ECM components, converting fatty streak into mature fibrofatty atheroma. Therefore, the migration of VSMCs plays a central role in the growth of AS lesions. Similar to their proliferation, the migration of VSMCs is regulated by growth promoters and inhibitors. PDGF-BB is one of the most potent stimuli for the migration of VSMCs. VSMCs form a structure known as podosomes when they migrate and invade. In fact, the proliferation and migration of VSMCs are closely associated. An increasing number of studies have found that miRs are involved in this process (Table III and Fig. 1).

\section{9. miRNAs promote the migration of VSMCs}

As mentioned earlier, miR-26a has multiple effects on VSMC functions. We have discussed its role in phenotypic regulation and proliferation. miR-26a can also promote the migration of VSMCs. As previously shown, cells deficient in miR-26a are 
Table III. miRs involved in the regulation of VSMC migration.

\begin{tabular}{lllc}
\hline Function & \multicolumn{1}{c}{ miRs } & \multicolumn{1}{c}{ Targets } & (Refs.) \\
\hline Promote & miR-26a & SMAD1/4 & $(26)$ \\
migration & miR-29b & DNMT3b & $(52)$ \\
& miR-133 & Sp-1 & $(17)$ \\
Inhibit & miR-143/145 & KLF5, ELK1, \\
migration & & myocardin & $(41)$ \\
& miR-181a & - & \\
& miR-195 & Cdc42, CCND1, & $(46)$ \\
& miR-638 & FGF1 & NDR1 \\
& Let-7g & LOX-1
\end{tabular}

miRs, microRNAs; VSMCs, vascular smooth muscle cells; DNMT3b, DNA methyltransferase 3b; Sp-1, specificity protein-1; KLF5, Krüppel-like factor 5; LOX-1, lectin-like oxidized low-density lipoprotein receptor-1.

Table IV. miRs involved in the regulation of VSMC apoptosis, ECM synthesis and calcification.

\begin{tabular}{lllc}
\hline Function & \multicolumn{1}{c}{ miRs } & Targets & (Refs.) \\
\hline $\begin{array}{l}\text { Inhibit } \\
\text { apoptosis }\end{array}$ & miR-21 & PDCD4, Sp-1 & $(30-32)$ \\
miR-26a & SMAD1/4 & $(26)$ \\
ECM synthesis & miR-145 & - & $(60)$ \\
$\begin{array}{l}\text { Inhibited } \\
\text { calcification }\end{array}$ & miR-29a/b & ADAMTS-7 & $(62)$ \\
\hline
\end{tabular}

miRs, microRNAs; VSMCs, vascular smooth muscle cells; ECM, extracellular matrix; PDCD4, programmed cell death protein 4, Sp-1, specificity protein-1.

less able to migrate towards a growth factor/serum gradient compared with the control group (45).

The miR-29 family (i.e., miR-29a, miR-29b and miR-29c) has been reported to be overexpressed in AS-related diseases (95,96). More specifically, miR-29b expression is upregulated in VSMCs treated with ox-LDL. miR-29b promotes VSMC migration by directly targeting DNA methyltransferase $3 b$ (DNMT3b) and epigenetically regulating the expression of MMP-2/MMP-9 genes (97). The MMP-2 and MMP-9 genes, which are involved in ECM remodeling, can promote VSMC migration and contribute to neointima formation (98).

\section{0. miRNAs inhibit the migration of VSMCs}

As mentioned earlier, the overexpression of miR-133 results in decreased VSMC proliferation and migration, while anti-miR-133 can lead to an increased proliferation and migration (32).
miR-143/145 inhibit podosome formation, which is an important morphological feature of VSMC migration in vitro $(99,100)$. miR-143/145 are downregulated in VSMCs treated with PDGF-BB, which has been proven to induce VSMC migration through podosome formation $(42,72)$.

As mentioned above, miR-181a inhibits the adhesion of VSMCs to collagen in response to Ang-II, which is essential for migration and proliferation (77). miR-195 can inhibit VSMC proliferation, migration and the synthesis of IL-1 $\beta$, IL-6 and IL- 8 induced by ox-LDL (78). miR-638, which is significantly downregulated in proliferative VSMCs, inhibits VSMC migration in response to PDGF-BB by targeting the NOR1/cyclin D1 pathway (87). Let-7g can significantly inhibit the oxLDL-induced LOX-1 and OCT-1 expression in VSMCs, as well as their proliferation and migration (101).

\section{1. miRNAs regulate VSMC apoptosis}

Apoptosis, or programmed cell death, is a part of normal development, senescence and other biological processes. The apoptosis of VSMCs in the fibrous cap causes the thinning of the fibrous cap and the expansion of the underlying necrotic core. Therefore, the apoptosis of VSMCs plays a critical role in determining plaque stability and thus, the most important consequence of AS, i.e., plaque rupture. The apoptosis of VSMCs is a tightly regulated process that contributes to atherosclerotic plaque rupture and restenosis (102). Myocardial infarction mainly occurs due to the uneven thinning and rupture of the fibrous cap and is often initiated at the shoulders of the lesion where cell apoptosis may occur (103). The rupture sites of the atherosclerotic plaque lack VSMCs and are rich in macrophages and inflammatory cells (104). The apoptosis of VSMCs has been detected in the shoulder regions of atherosclerotic plaques, which are most likely to rupture (105). VSMC apoptosis may be a potential critical target for the development of therapies for unstable AS. However, studies on the role of of miRs in the regulation of VSMC apoptosis in AS are relatively limited (Table IV).

In addition to its role in regulating phenotypic changes and the proliferation of VSMCs, miR-21 has an anti-apoptotic effect in cultured VSMCs. The inhibition of miR-21 has been proven to increase apoptosis in cultured VSMCs, as well as in vivo in injured rat carotid arteries (53). Phosphatase and tensin homolog (PTEN) and Bcl-2 are targets of miR-21. PTEN has been proven to regulate VSMC apoptosis through the inhibition of Akt phosphorylation/activation (106). Bcl-2, an anti-apoptotic protein, inhibits apoptosis by preventing the release of cytochrome $c$ from the mitochondria and plays a role in the initiation and progression of vascular cell apoptosis (107).

In addition to the regulation of the phenotype switch, and the proliferation and migration of VSMCs, miR-26a can also regulate the apoptosis of VSMCs. Cells deficient in miR-26a display significantly increased rates of apoptosis, indicating that miR-26a may inhibit the apoptosis of VSMCs (45). miR-26a has several possible target genes associated with apoptosis, incuding Bcl-2-antagonist/killer 1 (BAK1), p21 protein (Cdc42/Rac)-activated kinase 2 (PAK2) and sulfatase 1 (SULF1). However, further studies are is required to specify its target gene. 


\section{2. miRNAs regulate ECM synthesis by VSMCs}

Under pathological conditions, VSMCs may undergo the phenotypic change from the normal contractile state to a synthetic state, in which the cells an increased ability to migrate, proliferate and produce ECM proteins. Synthetic VSMCs can synthesize ECM conssting of fibrillar collagens and elastins and matrix-modifying enzymes that remodel the ECM. ECM production and remodeling are necessary to form the neointima (108). However, studies on the effects of miRs on ECM synthesis by VSMCs are limited (Table IV).

Lysyl oxidase (Lox), which can crosslink adjacent collagen triple helices, confer tensile strength to collagen fibrils and contribute to neointimal growth through the chemotactic activity of VSMCs and monocytes $(109,110)$, is negatively regulated by miR-145. ApoE, which can inhibit ECM gene expression, reduces the levels of Lox by increasing the expression of miR-145 (111).

\section{3. miRNAs regulate VSMC calcification}

Vascular calcification is defined as the deposition of calcium phosphate mineral in the vessel wall. The exact role of vascular calcification in plaque stability remains unclear. VSMCs have been proven to significantly contribute to vascular calcification, which is a prominent feature of AS (112). miRs have been reported to be involved in the regulation of VSMC calcification (Table IV).

In addition to the role of miR-29b in promoting VSMC migration, miR-29a/b is downregulated in calcifying VSMCs. miR-29a/b inhibits VSMC calcification by suppressing the expression of a disintegrin and metalloproteinase with thrombospondin motifs-7 (ADAMTS-7) (113). In injured vessels, ADAMTS-7 has been proven to mediate the degradation of cartilage oligomeric matrix protein (COMP) $(114,115)$, which can inhibit VSMC calcification in vitro and in vivo by interfering with BMP 2 expression and preventing the osteochondrogenic transdifferentiation of VSMCs (116).

miR-125b is significantly downregulated in calcified vessels. miR-125b expression may be increased during atherosclerotic inflammation processes since an increased expression of miR-125b has been observed in $\mathrm{ApoE}^{-/-}$mice fed a high-fat diet (117). Furthermore, the inhibition of endogenous miR-125b promotes the transdifferentiation of VSMCs into osteoblast-like cells. miR-125b has been proven to be involved in vascular calcification by targeting SP7 (osterix) (118). SP7 is a zinc finger transcription factor expressed by osteoblasts is necessary for osteoblast differentiation in mice (119).

\section{Implications in the management of AS}

The regulation of specific miRs during neointima formation in AS and restenosis suggests that miRs are key determinants of the changes in the function of VSMCs. Altered circulating levels of miRs in patients with AS may provide a novel approach for the detection of CAD. For example, as the circulating levels of miR-145 are lower in the serum of patients with CAD (33), it may serve as a biomarker for CAD. However, large-scale studies are required to confirm the use of miRs as biomarkers for the diagnosis of disease. Furthermore, miRs may also serve as potential targets for therapeutic strategies since their expression can be altered through genetic approaches. Since the modifications of miRs are reversible, it is possible to use specific miRs to reduce VSMC proliferation, migration and apoptosis, in order to prevent neointima formation in AS and restenosis. For example, the adenovirus-mediated gene transfer of miR-145 in rat balloon-injured carotid arteries has been proven to inhibit neointimal lesion formation. The knockdown of miR-221 and -222 in vessels reduces VSMC proliferation and intimal thickening in response to vascular injury (46). However, in vivo data on the role of miRs in the regulation of VSMC functions in AS and restenosis are limited. Further studies are required to elucidate the underlying mechanisms of action of miRs and their contribution to VSMC functions and AS in vivo.

\section{Conclusions}

In this review, we summarized the roles of miRs in the function of VSMCs and their contributions to AS. miRs critically affect VSMC functions, including differentiation, proliferation, migration, ECM synthesis, calcification and apoptosis, all of which play critical roles in the pathogenesis of AS and restenosis. We also discussed the changes in miR expression patterns in humans and animal models associated of AS. We focused on the different functions of VSMCs to elucidate the mechanisms through which miRs regulate each specific cellular function. miRs exert their functions by regulating a number of target genes, such as KLF4, p27 and Sp-1. The discovery of miR interference provides a promising insight into the understanding of the regulation of gene expression.

\section{References}

1. Lusis AJ: Atherosclerosis. Nature 407: 233-241, 2000.

2. Gittenberger-de Groot AC, DeRuiter MC, Bergwerff M and Poelmann RE: Smooth muscle cell origin and its relation to heterogeneity in development and disease. Arterioscler Thromb Vasc Biol 19: 1589-1594, 1999.

3. Frid MG, Dempsey EC, Durmowicz AG and Stenmark KR: Smooth muscle cell heterogeneity in pulmonary and systemic vessels. Importance in vascular disease. Arterioscler Thromb Vasc Biol 17: 1203-1209, 1997.

4. Schachter M: Vascular smooth muscle cell migration, atherosclerosis, and calcium channel blockers. Int J Cardiol 62 (Suppl 2): S85-S90, 1997.

5. Libby P, Sukhova G, Lee RT and Liao JK: Molecular biology of atherosclerosis. Int J Cardiol 62 (Suppl 2): S23-S29, 1997.

6. Schwartz SM: Smooth muscle migration in atherosclerosis and restenosis. J Clin Invest 100 (Suppl): S87-S89, 1997.

7. Doran AC, Meller N and McNamara CA: Role of smooth muscle cells in the initiation and early progression of atherosclerosis. Arterioscler Thromb Vasc Biol 28: 812-819, 2008.

8. Huntzinger E and Izaurralde E: Gene silencing by microRNAs: contributions of translational repression and mRNA decay. Nat Rev Genet 12: 99-110, 2011.

9. Cho WC: OncomiRs: the discovery and progress of microRNAs in cancers. Mol Cancer 6: 60, 2007.

10. Small EM and Olson EN: Pervasive roles of microRNAs in cardiovascular biology. Nature 469: 336-342, 2011.

11. Song Z and Li G: Role of specific microRNAs in regulation of vascular smooth muscle cell differentiation and the response to injury. J Cardiovasc Transl Res 3: 246-250, 2010.

12. Zernecke A: MicroRNAs in the regulation of immune cell functions-implications for atherosclerotic vascular disease. Thromb Haemost 107: 626-633, 2012.

13. Aghabozorg Afjeh SS and Ghaderian SM: The role of microRNAs in cardiovascular disease. Int J Mol Cell Med 2: 50-57, 2013. 
14. Robinson HC and Baker AH: How do microRNAs affect vascular smooth muscle cell biology? Curr Opin Lipidol 23: 405-411, 2012.

15. Owens GK, Kumar MS and Wamhoff BR: Molecular regulation of vascular smooth muscle cell differentiation in development and disease. Physiol Rev 84: 767-801, 2004.

16. Stegemann JP, Hong H and Nerem RM: Mechanical, biochemical, and extracellular matrix effects on vascular smooth muscle cell phenotype. J Appl Physiol (1985) 98: 2321-2327, 2005.

17. Tang Y, Yang X, Friesel RE, Vary CP and Liaw L: Mechanisms of TGF- $\beta$-induced differentiation in human vascular smooth muscle cells. J Vasc Res 48: 485-494, 2011.

18. Millette E, Rauch BH, Kenagy RD, Daum G and Clowes AW: Platelet-derived growth factor-BB transactivates the fibroblas growth factor receptor to induce proliferation in human smooth muscle cells. Trends Cardiovasc Med 16: 25-28, 2006.

19. Raines EW: PDGF and cardiovascular disease. Cytokine Growth Factor Rev 15: 237-254, 2004.

20. Ross R: The pathogenesis of atherosclerosis: a perspective for the 1990s. Nature 362: 801-809, 1993

21. Chen J, Yin H, Jiang Y, et al: Induction of microRNA-1 by myocardin in smooth muscle cells inhibits cell proliferation. Arterioscler Thromb Vasc Biol 31: 368-375, 2011.

22. Xie C, Huang H, Sun X, et al: MicroRNA-1 regulates smooth muscle cell differentiation by repressing Kruppel-like factor 4. Stem Cells Dev 20: 205-210, 2011.

23. Jiang $\mathrm{Y}$, Yin $\mathrm{H}$ and Zheng $\mathrm{XL}$ : MicroRNA-1 inhibits myocardin-induced contractility of human vascular smooth muscle cells. J Cell Physiol 225: 506-511, 2010.

24. Suzuki T, Aizawa K, Matsumura T and Nagai R: Vascular implications of the Kruppel-like family of transcription factors. Arterioscler Thromb Vasc Biol 25: 1135-1141, 2005.

25. Liu Y, Sinha S, McDonald OG, Shang Y, Hoofnagle MH and Owens GK: Kruppel-like factor 4 abrogates myocardin-induced activation of smooth muscle gene expression. J Biol Chem 280: 9719-9727, 2005.

26. Yoshida T, Kaestner KH and Owens GK: Conditional deletion of Kruppel-like factor 4 delays downregulation of smooth muscle cell differentiation markers but accelerates neointimal formation following vascular injury. Circ Res 102: 1548-1557, 2008.

27. King KE, Iyemere VP, Weissberg PL and Shanahan CM: Kruppel-like factor 4 (KLF4/GKLF) is a target of bone morphogenetic proteins and transforming growth factor beta 1 in the regulation of vascular smooth muscle cell phenotype. J Biol Chem 278: 11661-11669, 2003.

28. Wang C, Han M, Zhao XM and Wen JK: Kruppel-like factor 4 is required for the expression of vascular smooth muscle cell differentiation marker genes induced by all-trans retinoic acid. J Biochem 144: 313-321, 2008

29. Katakami N, Kaneto H, Hao H, et al: Role of pim-1 in smooth muscle cell proliferation. J Biol Chem 279: 54742-54749, 2004.

30. Wang J, Yan CH, Li Y, et al: MicroRNA-31 controls phenotypic modulation of human vascular smooth muscle cells by regulating its target gene cellular repressor of E1A-stimulated genes. Exp Cell Res 319: 1165-1175, 2013 .

31. Han Y, Deng J, Guo L, et al: CREG promotes a mature smooth muscle cell phenotype and reduces neointimal formation in balloon-injured rat carotid artery. Cardiovasc Res 78: 597-604, 2008.

32. Torella D, Iaconetti C, Catalucci D, et al: MicroRNA-133 controls vascular smooth muscle cell phenotypic switch in vitro and vascular remodeling in vivo. Circ Res 109: 880-893, 2011.

33. Fichtlscherer S, De Rosa S, Fox H, et al: Circulating microRNAs in patients with coronary artery disease. Circ Res 107: 677-684, 2010.

34. Miano JM: Serum response factor: toggling between disparate programs of gene expression. J Mol Cell Cardiol 35: 577-593, 2003.

35. Madsen CS, Hershey JC, Hautmann MB, White SL and Owens GK: Expression of the smooth muscle myosin heavy chain gene is regulated by a negative-acting GC-rich element located between two positive-acting serum response factor-binding elements. J Biol Chem 272: 6332-6340, 1997.

36. Deaton RA, Gan Q and Owens GK: Sp1-dependent activation of KLF4 is required for PDGF-BB-induced phenotypic modulation of smooth muscle. Am J Physiol Heart Circ Physiol 296: H1027-H1037, 2009.

37. Cordes KR, Sheehy NT, White MP, et al: miR-145 and miR-143 regulate smooth muscle cell fate and plasticity. Nature 460: 705-710, 2009
38. Raitoharju E, Lyytikäinen LP, Levula M, et al: miR-21, miR-210, miR-34a, and miR-146a/b are upregulated in human atherosclerotic plaques in the Tampere Vascular Study. Atherosclerosis 219: 211-217, 2011.

39. Hergenreider E, Heydt S, Tréguer K, et al: Atheroprotective communication between endothelial cells and smooth muscle cells through miRNAs. Nat Cell Biol 14: 249-256, 2012.

40. Lovren F, Pan Y, Quan A, et al: MicroRNA-145 targeted therapy reduces atherosclerosis. Circulation 126 (Suppl 1): S81-S90, 2012.

41. Boettger T, Beetz N, Kostin S, et al: Acquisition of the contractile phenotype by murine arterial smooth muscle cells depends on the Mir143/145 gene cluster. J Clin Invest 119: 2634-2647, 2009.

42. Rangrez AY, Massy ZA, Metzinger-Le Meuth V and Metzinger L: miR-143 and miR-145: molecular keys to switch the phenotype of vascular smooth muscle cells. Circ Cardiovasc Genet 4: 197-205, 2011.

43. Chan MC, Hilyard AC, Wu C, et al: Molecular basis for antagonism between PDGF and the TGFbeta family of signalling pathways by control of miR-24 expression. EMBO J 29: 559-573, 2010.

44. Chan MC, Nguyen PH, Davis BN, et al: A novel regulatory mechanism of the bone morphogenetic protein (BMP) signaling pathway involving the carboxyl-terminal tail domain of BMP type II receptor. Mol Cell Biol 27: 5776-5789, 2007.

45. Leeper NJ, Raiesdana A, Kojima Y, et al: MicroRNA-26a is a novel regulator of vascular smooth muscle cell function. J Cell Physiol 226: 1035-1043, 2011

46. Liu X, Cheng Y, Zhang S, Lin Y, Yang J and Zhang C: A necessary role of miR-221 and miR-222 in vascular smooth muscle cell proliferation and neointimal hyperplasia. Circ Res 104: 476-487, 2009.

47. Davis BN, Hilyard AC, Nguyen PH, Lagna G and Hata A: Induction of microRNA-221 by platelet-derived growth factor signaling is critical for modulation of vascular smooth muscle phenotype. J Biol Chem 284: 3728-3738, 2009.

48. Liu X, Cheng Y, Yang J, Xu L and Zhang C: Cell-specific effects of miR-221/222 in vessels: molecular mechanism and therapeutic application. J Mol Cell Cardiol 52: 245-255, 2012.

49. Tanner FC, Yang ZY, Duckers E, Gordon D, Nabel GJ and Nabel EG: Expression of cyclin-dependent kinase inhibitors in vascular disease. Circ Res 82: 396-403, 1998.

50. Stewart MC, Kadlcek RM, Robbins PD, MacLeod JN and Ballock RT: Expression and activity of the CDK inhibitor p57Kip2 in chondrocytes undergoing hypertrophic differentiation. J Bone Miner Res 19: 123-132, 2004.

51. Ashman LK: The biology of stem cell factor and its receptor c-kit. Int J Biochem Cell Biol 31: 1037-1051, 1999.

52. Yang G, Pei Y, Cao Q and Wang R: MicroRNA-21 represses human cystathionine gamma-lyase expression by targeting at specificity protein-1 in smooth muscle cells. J Cell Physiol 227: 3192-3200, 2012

53. Ji R, Cheng Y, Yue J, et al: MicroRNA expression signature and antisense-mediated depletion reveal an essential role of microRNA in vascular neointimal lesion formation. Circ Res 100: 1579-1588, 2007.

54. Davis BN, Hilyard AC, Lagna G and Hata A: SMAD proteins control DROSHA-mediated microRNA maturation. Nature 454: 56-61, 2008

55. Ozpolat B, Akar U, Steiner M, et al: Programmed cell death-4 tumor suppressor protein contributes to retinoic acid-induced terminal granulocytic differentiation of human myeloid leukemia cells. Mol Cancer Res 5: 95-108, 2007.

56. Cash AC and Andrews J: Fine scale analysis of gene expression in Drosophila melanogaster gonads reveals programmed cell death 4 promotes the differentiation of female germline stem cells. BMC Dev Biol 12: 4, 2012

57. Curcio A, Torella D and Indolfi C: Mechanisms of smooth muscle cell proliferation and endothelial regeneration after vascular injury and stenting: approach to therapy. Circ J 75: 1287-1296, 2011.

58. Liu X, Cheng Y, Chen $\mathrm{X}$, Yang J, Xu L and Zhang C: MicroRNA-31 regulated by the extracellular regulated kinase is involved in vascular smooth muscle cell growth via large tumor suppressor homolog 2. J Biol Chem 286: 42371-42380, 2011.

59. An Y, Kang Q, Zhao Y, Hu X and Li N: Lats2 modulates adipocyte proliferation and differentiation via hippo signaling. PloS One 8: e72042, 2013

60. Wu WH, Hu CP, Chen XP, et al: MicroRNA-130a mediates proliferation of vascular smooth muscle cells in hypertension. Am J Hypertens 24: 1087-1093, 2011. 
61. Weir L, Chen D, Pastore C, Isner JM and Walsh K: Expression of gax, a growth arrest homeobox gene, is rapidly downregulated in the rat carotid artery during the proliferative response to balloon injury. J Biol Chem 270: 5457-5461, 1995.

62. Xia S, Tai X, Wang Y, et al: Involvement of Gax gene in hypoxia-induced pulmonary hypertension, proliferation, and apoptosis of arterial smooth muscle cells. Am J Respir Cell Mol Biol 44: 66-73, 2011.

63. Saito T, Itoh H, Yamashita J, et al: Angiotensin II suppresses growth arrest specific homeobox (Gax) expression via redox-sensitive mitogen-activated protein kinase (MAPK). Regul Pept 127: 159-167, 2005

64. Gorski DH, LePage DF, Patel CV, Copeland NG, Jenkins NA and Walsh K: Molecular cloning of a diverged homeobox gene that is rapidly downregulated during the G0/G1 transition in vascular smooth muscle cells. Mol Cell Biol 13: 3722-3733, 1993.

65. Yamashita J, Itoh H, Ogawa Y, et al: Opposite regulation of Gax homeobox expression by angiotensin II and C-type natriuretic peptide. Hypertension 29: 381-387, 1997.

66. Witzenbichler B, Kureishi Y, Luo Z, Le Roux A, Branellec D and Walsh K: Regulation of smooth muscle cell migration and integrin expression by the Gax transcription factor. J Clin Invest 104: 1469-1480, 1999.

67. Wassmann S, Wassmann K, Jung A, et al: Induction of p53 by GKLF is essential for inhibition of proliferation of vascular smooth muscle cells. J Mol Cell Cardiol 43: 301-307, 2007.

68. Sun SG, Zheng B, Han M, et al: miR-146a and Krüppel-like factor 4 form a feedback loop to participate in vascular smooth muscle cell proliferation. EMBO Rep 12: 56-62, 2011.

69. Zhang Y, Wang Y, Wang X, et al: Insulin promotes vascular smooth muscle cell proliferation via microRNA-208-mediated downregulation of p21. J Hypertens 29: 1560-1568, 2011.

70. Zheng X, Li A, Zhao L, et al: Key role of microRNA-15a in the KLF4 suppressions of proliferation and angiogenesis in endothelial and vascular smooth muscle cells. Biochem Biophys Res Commun 437: 625-631, 2013.

71. Cheng Y, Liu X, Yang J, et al: MicroRNA-145, a novel smooth muscle cell phenotypic marker and modulator, controls vascular neointimal lesion formation. Circ Res 105: 158-166, 2009.

72. Quintavalle M, Elia L, Condorelli G and Courtneidge SA: MicroRNA control of podosome formation in vascular smooth muscle cells in vivo and in vitro. J Cell Biol 189: 13-22, 2010.

73. Wang YS, Chou WW, Chen KC, Cheng HY, Lin RT and Juo SH: MicroRNA-152 mediates DNMT1-regulated DNA methylation in the estrogen receptor $\alpha$ gene. PloS One 7: e30635, 2012.

74. Li E, Bestor TH and Jaenisch R: Targeted mutation of the DNA methyltransferase gene results in embryonic lethality. Cell 69: 915-926, 1992.

75. Huang RS, Hu GQ, Lin B, Lin ZY and Sun CC: MicroRNA-155 silencing enhances inflammatory response and lipid uptake in oxidized low-density lipoprotein-stimulated human THP-1 macrophages. J Investig Med 58: 961-967, 2010.

76. Ma X, Ma C and Zheng X: MicroRNA-155 in the pathogenesis of atherosclerosis: a conflicting role? Heart Lung Circ 22: 811-818, 2013.

77. Remus EW, Lyle AN, Weiss D, et al: miR181a protects against angiotensin II-induced osteopontin expression in vascular smooth muscle cells. Atherosclerosis 228: 168-174, 2013

78. Wang YS, Wang HY, Liao YC, et al: MicroRNA-195 regulates vascular smooth muscle cell phenotype and prevents neointimal formation. Cardiovasc Res 95: 517-526, 2012.

79. Ammit AJ and Panettieri RA Jr: Invited review: the circle of life: cell cycle regulation in airway smooth muscle. J Appl Physiol (1985) 91: 1431-1437, 2001.

80. Chotani MA, Touhalisky K and Chiu IM: The small GTPases Ras, Rac, and Cdc42 transcriptionally regulate expression of human fibroblast growth factor 1. J Biol Chem 275: 30432-30438, 2000.

81. Lindner V and Reidy MA: Proliferation of smooth muscle cells after vascular injury is inhibited by an antibody against basic fibroblast growth factor. Proc Natl Acad Sci USA 88: 3739-3743, 1991.

82. Hanna AK, Fox JC, Neschis DG, Safford SD, Swain JL and Golden MA: Antisense basic fibroblast growth factor gene transfer reduces neointimal thickening after arterial injury. J Vasc Surg 25: 320-325, 1997.

83. Merlet E, Atassi F, Motiani RK, et al: miR-424/322 regulates vascular smooth muscle cell phenotype and neointimal formation in the rat. Cardiovasc Res 98: 458-468, 2013.

84. Liu Q, Fu H, Sun F, et al: miR-16 family induces cell cycle arrest by regulating multiple cell cycle genes. Nucleic acids Res 36: 5391-5404, 2008 .
85. Sahoo SK, Kim T, Kang GB, Lee JG, Eom SH and Kim do H: Characterization of calumenin-SERCA2 interaction in mouse cardiac sarcoplasmic reticulum. J Biol Chem 284: 31109-31121, 2009.

86. Sun Y, Chen D, Cao L, et al: miR-490-3p modulates the proliferation of vascular smooth muscle cells induced by ox-LDL through targeting PAPP-A. Cardiovasc Res 100: 272-279, 2013.

87. Li P, Liu Y, Yi B, et al: MicroRNA-638 is highly expressed in human vascular smooth muscle cells and inhibits PDGF-BB-induced cell proliferation and migration through targeting orphan nuclear receptor NOR1. Cardiovasc Res 99: 185-193, 2013

88. Bonta PI, Pols TW and de Vries CJ: NR4A nuclear receptors in atherosclerosis and vein-graft disease. Trends Cardiovasc Med 17: 105-111, 2007.

89. Yu ML, Wang JF, Wang GK, et al: Vascular smooth muscle cell proliferation is influenced by let-7d microRNA and its interaction with KRAS. Circ J 75: 703-709, 2011.

90. Doe Z, Fukumoto Y, Takaki A, et al: Evidence for Rho-kinase activation in patients with pulmonary arterial hypertension. Circ J 73: 1731-1739, 2009.

91. Zhou YC and WaxmanDJ: Cross-talk between januskinase-signal transducer and activator of transcription (JAK-STAT) and peroxisome proliferator-activated receptor-alpha (PPARalpha) signaling pathways. Growth hormone inhibition of pparalpha transcriptional activity mediated by stat $5 \mathrm{~b}$. J Biol Chem 274: 2672-2681, 1999.

92. Chen KC, Hsieh IC, Hsi E, et al: Negative feedback regulation between microRNA let-7g and the oxLDL receptor LOX-1. J Cell Sci 124: 4115-4124, 2011.

93. Mehta JL, Chen J, Hermonat PL, Romeo F and Novelli G: Lectin-like, oxidized low-density lipoprotein receptor-1 (LOX-1): a critical player in the development of atherosclerosis and related disorders. Cardiovasc Res 69: 36-45, 2006.

94. Mehta JL and Li DY: Identification and autoregulation of receptor for OX-LDL in cultured human coronary artery endothelial cells. Biochem Biophys Res Commun 248: 511-514, 1998.

95. Tan KS, Armugam A, Sepramaniam S, et al: Expression profile of MicroRNAs in young stroke patients. PloS One 4: e7689, 2009.

96. Urbich C, Kuehbacher A and Dimmeler S: Role of microRNAs in vascular diseases, inflammation, and angiogenesis. Cardiovasc Res 79: 581-588, 2008

97. Chen KC, Wang YS, Hu CY, et al: OxLDL upregulates microRNA-29b, leading to epigenetic modifications of MMP-2/MMP-9 genes: a novel mechanism for cardiovascular diseases. FASEB J 25: 1718-1728, 2011.

98. Aoyagi M, Yamamoto M, Azuma H, et al: Immunolocalization of matrix metalloproteinases in rabbit carotid arteries after balloon denudation. Histochem Cell Biol 109: 97-102, 1998.

99. Gimona M, Kaverina I, Resch GP, Vignal E and Burgstaller G: Calponin repeats regulate actin filament stability and formation of podosomes in smooth muscle cells. Mol Biol Cell 14: 2482-2491, 2003.

100. Linder S and Aepfelbacher M: Podosomes: adhesion hot-spots of invasive cells. Trends Cell Biol 13: 376-385, 2003.

101.Kunugi S, Iwabuchi S, Matsuyama D, Okajima T and Kawahara K: Negative-feedback regulation of ATP release: ATP release from cardiomyocytes is strictly regulated during ischemia. Biochem Biophys Res Commun 416: 409-415, 2011.

102. Bennett MR: Apoptosis of vascular smooth muscle cells in vascular remodelling and atherosclerotic plaque rupture. Cardiovasc Res 41: 361-368, 1999.

103. Fuster V: Lewis A. Conner Memorial Lecture. Mechanisms leading to myocardial infarction: insights from studies of vascular biology. Circulation 90: 2126-2146, 1994

104. Davies MJ, Richardson PD, Woolf N, Katz DR and Mann J: Risk of thrombosis in human atherosclerotic plaques: role of extracellular lipid, macrophage, and smooth muscle cell content. Br Heart J 69: 377-381, 1993.

105. Geng YJ and Libby P: Evidence for apoptosis in advanced human atheroma. Colocalization with interleukin-1 beta-converting enzyme. Am J Pathol 147: 251-266, 1995

106. Sedding DG, Widmer-Teske R, Mueller A, et al: Role of the phosphatase PTEN in early vascular remodeling. PloS One 8: e55445, 2013

107. Geng YJ: Molecular signal transduction in vascular cell apoptosis. Cell Res 11: 253-264, 2001.

108. Clowes AW, Clowes MM, Fingerle J and Reidy MA: Regulation of smooth muscle cell growth in injured artery. J Cardiovasc Pharmacol 14 (Suppl 6): S12-S15, 1989. 
109.Li W, Liu G, Chou IN and Kagan HM: Hydrogen peroxide-mediated, lysyl oxidase-dependent chemotaxis of vascular smooth muscle cells. J Cell Biochem 78: 550-557, 2000.

110. Lazarus HM, Cruikshank WW, Narasimhan N, Kagan HM and Center DM: Induction of human monocyte motility by lysyl oxidase. Matrix Biol 14: 727-731, 1995

111. Kothapalli D, Liu SL, Bae YH, et al: Cardiovascular protection by ApoE and ApoE-HDL linked to suppression of ECM gene expression and arterial stiffening. Cell Rep 2: 1259-1271, 2012.

112. Iyemere VP, Proudfoot D, Weissberg PL and Shanahan CM: Vascular smooth muscle cell phenotypic plasticity and the regulation of vascular calcification. J Intern Med 260: 192-210, 2006.

113. Du Y, Gao C, Liu Z, et al: Upregulation of a disintegrin and metalloproteinase with thrombospondin motifs- 7 by miR-29 repression mediates vascular smooth muscle calcification. Arterioscler Thromb Vasc Biol 32: 2580-2588, 2012.

114. Wang L, Zheng J, Bai X, et al: ADAMTS-7 mediates vascular smooth muscle cell migration and neointima formation in balloon-injured rat arteries. Circ Res 104: 688-698, 2009.
115. Liu CJ, Kong W, Ilalov K, et al: ADAMTS-7: a metalloproteinase that directly binds to and degrades cartilage oligomeric matrix protein. FASEB J 20: 988-990, 2006.

116. Du Y, Wang Y, Wang L, et al: Cartilage oligomeric matrix protein inhibits vascular smooth muscle calcification by interacting with bone morphogenetic protein-2. Circ Res 108: 917-928, 2011.

117. Villeneuve LM, Kato M, Reddy MA, Wang M, Lanting L and Natarajan R: Enhanced levels of microRNA-125b in vascular smooth muscle cells of diabetic $\mathrm{db} / \mathrm{db}$ mice lead to increased inflammatory gene expression by targeting the histone methyltransferase Suv39h1. Diabetes 59: 2904-2915, 2010.

118. Goettsch C, Rauner M, Pacyna N, Hempel U, Bornstein SR and Hofbauer LC: miR-125b regulates calcification of vascular smooth muscle cells. Ame J Pathol 179: 1594-1600, 2011.

119. Zhu F, Friedman MS, Luo W, Woolf P and Hankenson KD: The transcription factor osterix (SP7) regulates BMP6-induced human osteoblast differentiation. J Cell Physiol 227: 2677-2685, 2012. 